\title{
VARIATION OF MAGNETIC FIELDS AND FLOWS DURING THE SOLAR CYCLE
}

\author{
A. Brandenburg and I. Tuominen \\ Observatory and Astrophysics Laboratory, University of Helsinki \\ Tähtitorninmäki, SF-00130 Helsinki, Finland
}

\begin{abstract}
We have studied dynamo models with realistic assumptions for the induction effects and compare the resulting magnetic fields with observations. We make use of recent results of helioseismology to infer the differential rotation and adopt mixing length approach to get the form of the $\alpha$-effect, which is in general anisotropic. Dynamo models are computed numerically using an eigenvalue method. Finally, we discuss the resulting Lorentz force, which is relevant for explaining observed flows such as the torsional waves, as a back reaction of the dynamo magnetic fields. Comparison is made in particular with Mt.Wilson/Kitt Peak magnetograms and synoptic charts showing the magnetic flux, torsional oscillation pattern, and the distribution of ephemeral regions, coronal activity, and polar faculae.
\end{abstract}

\section{INTRODUCTION}

The first solar $\alpha \Omega$-dynamo models by Steenbeck and Krause / $1 /$ showed many observed features of the solar activity cycle, for example the periodic migration of sunspot belts towards the equator, Hale's polarity rule for bipolar spots on $\mathrm{N}$ - and $\mathrm{S}$-hemisphere, and the phase relation between poloidal and toroidal field components $/ 2,3 /$. Nevertheless, many problems of dynamo theory have been pointed out during the last decade. In particular the intermittent structure of magnetic fields in the convection zone leads to buoyancy effects, which can transport the magnetic flux to the surface on a time scale much shorter than the solar cycle. Other problems concern the strength of the coefficients for the $\alpha$-effect and for turbulent diffusion, and the question of calculated periods, which are much shorter than the solar cycle. This has led to the impression that dynamo theory fails altogether to explain the solar activity cycle /15/. However, well developed alternative theories do not exist.

In the kinematic (linear) mean field approach, differential rotation and $\alpha$-effect are assumed to be known. This gave rise to a variety of quite different models in the past. Due to recently improved determinations of the solar internal angular velocity from helioseismology, it is necessary to reconsider the $\alpha \Omega$-dynamos. The goal of the present paper is to present more realistic models based on these new observational constraints.

\section{KINEMATIC DYNAMO THEORY}

We assume that the mean magnetic field $B$ of the Sun is generated by an $\alpha \Omega$-dynamo mechanism, which is described by the induction equation:

$$
\frac{\partial B}{\partial t}=\operatorname{curl}(\boldsymbol{u} \times \boldsymbol{B}+\mathcal{E}-\eta \operatorname{curl} B)
$$

where $\eta$ is the molecular magnetic diffusivity. Turbulent motions in the solar convection zone give rise to an additional electromotive force $\mathcal{E}=\left\langle\boldsymbol{u}^{\prime} \times \boldsymbol{B}^{\prime}\right\rangle$ (e.g. $\alpha$-effect). The differential rotation $\Omega(r, \theta)$, which enters into Eq.(1) via $\boldsymbol{u}=\boldsymbol{r} \times \Omega \hat{\boldsymbol{z}}$, is important for generating toroidal field from a poloidal one. The function $\Omega(r, \theta)$ is observed to some extent by means of helioseismology. In Fig.1 we have combined data obtained from different authors. 


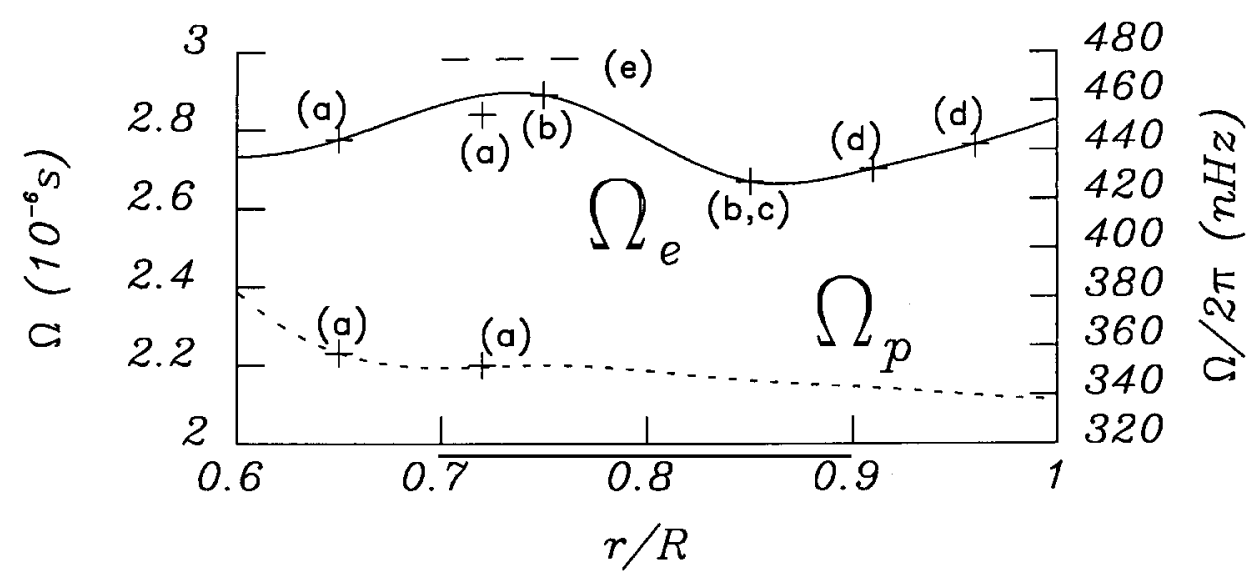

Figure 1: The internal solar angular velocity at the equator $\Omega_{e}(r)$ and the poles $\Omega_{p}(r)$, inferred from results of helioseismology. The references are: (a) Brown et al. $/ 6 /$, (b) Duvall and Harvey $/ 7 /$ (their Fig.4a), (c) Hill $/ 8 /$ (his Fig.2), (d) Hill et al. /9/ (their Fig.2). The dashed line (e) at $475 \mathrm{nHz}$ denotes the angular velocity of very young sunspots $/ 17 /$.

An expression for $\mathcal{E}$ in the presence of weakly inhomogeneous turbulence has been derived in the frame-work of first order smoothing /4,5/:

$$
\mathcal{E}=-\eta_{t} \operatorname{curl} \boldsymbol{B}-\gamma \hat{\boldsymbol{r}} \times \boldsymbol{B}+\beta \hat{\boldsymbol{z}} \times \operatorname{curl} \boldsymbol{B}+\alpha_{1}(\hat{\boldsymbol{r}} \cdot \hat{\boldsymbol{z}}) \boldsymbol{B}+\alpha_{4}[(\boldsymbol{B} \cdot \hat{\boldsymbol{r}}) \hat{\boldsymbol{z}}+(\boldsymbol{B} \cdot \hat{\boldsymbol{z}}) \hat{\boldsymbol{r}}]+\ldots
$$

where $\eta_{t}, \gamma, \beta, \alpha_{1}$, and $\alpha_{4}$ are certain functions of radius $r$. In the following we shall employ rough estimations in terms of correlation time $\tau$ and turbulent r.m.s. velocity $V$ (see $/ 4,5,10,11 /$ ). For the turbulent magnetic diffusivity $\eta_{t}$ and the isotropic $\alpha$-effect one obtains:

$$
\begin{gathered}
\eta_{t} \approx \frac{1}{3} \tau V^{2} \\
\alpha_{1} \approx-\frac{8}{15} \Omega \rho^{-2} \frac{d}{d r}\left(\rho^{2} \tau^{2} V^{2}\right) .
\end{gathered}
$$

The diamagnetic effect is given by $\gamma=\frac{1}{2} d \eta_{t} / d r$ and for the so-called $\Omega \times J$-effect we take $\beta \approx \frac{4}{15} \Omega \tau^{2} V^{2}$ and finally $\alpha_{4}=-\alpha_{1} / 4$ (see $/ 5 /$ ). In the mixing length approach one assumes usually $\tau=C_{r} \ell / V$, where $\ell$ is the mixing length and $C_{\tau}$ a factor of order unity. However, this expression becomes singular at the bottom of the convection zone $(V \rightarrow 0)$ and we shall therefore replace it by a linear extrapolation for $r \leq 0.85 R . C_{\tau}$ will be specified later. In Fig.2 we have plotted $\eta_{t}$ and $\alpha_{1}$ using $V$ and $\ell$ from a solar mixing length model.
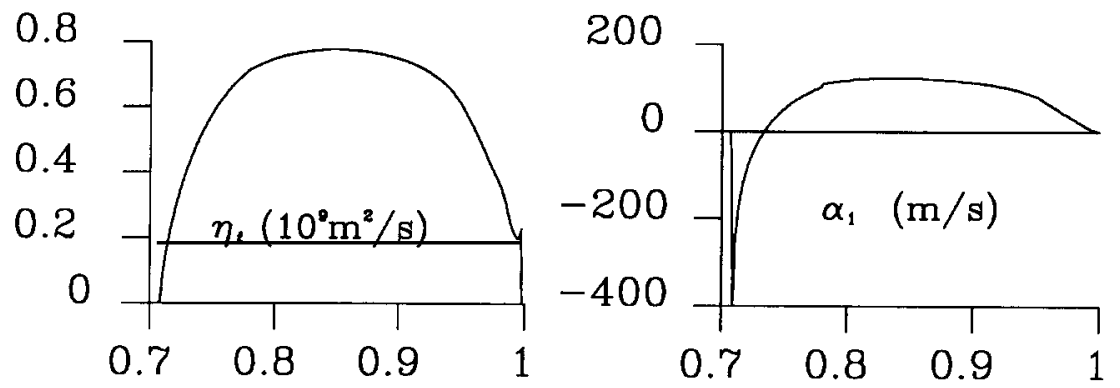

Figure 2: $\eta_{t}$ and $\alpha_{1}$ using $V$ and $\ell$ from a solar mixing length model $\left(C_{\tau}=1\right)$.

\section{SOLAR DYNAMO MODELS}

We have computed dynamo models using the functions $\Omega_{e / p}, \alpha_{1}$, etc. described in the previous section. As is usual in the linear dynamo theory, one is interested in the marginal solution, which is neither growing nor decaying. This can be achieved by scaling $\alpha_{1,4}$ and $\beta$ with a factor C. Using $C=0.05$ and $C_{\tau}=0.1$ we found an oscillatory dynamo solution with a magnetic period of 15 years. In Figure 3 we have plotted the field geometry for this axisymmetrix dipole type mode (A0, see ref./11/): The most striking feature in 


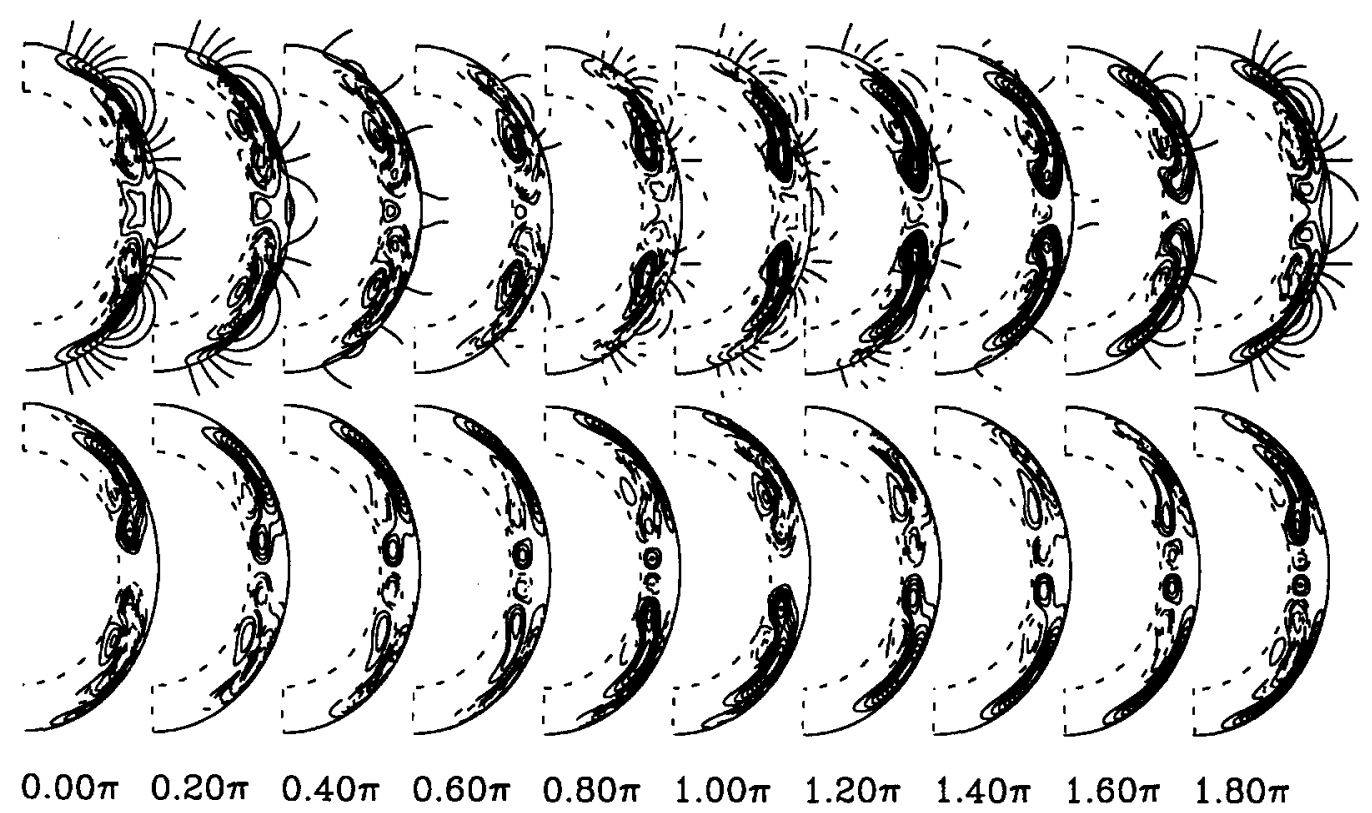

Figure 3: The evolution of the axisymmetrix dipole-type mode of a solar dynamo model. In the upper half are plotted cross-sections showing the fieldlines of the poloidal component, and in the lower one, lines of constant toroidal field.

Figure 3 is the clear separation between two differently propagating dynamo waves, which was already found by Makarov et al. $/ 12 /$ for a much simpler model using a WKB approximation. During the time interval $\omega t=0 \ldots 0.6 \pi(2 \pi / \omega=$ cycle period $)$ new magnetic field is generated at $\approx 45^{\circ}$ and is quickly amplified. From $\omega t=1 \ldots 1.6 \pi$ part of the field migrates down to the equator while in the upper layers the main sense of propagation is towards the poles. This becomes more evident from a generalized butterfly diagram, shown in Figure 4.

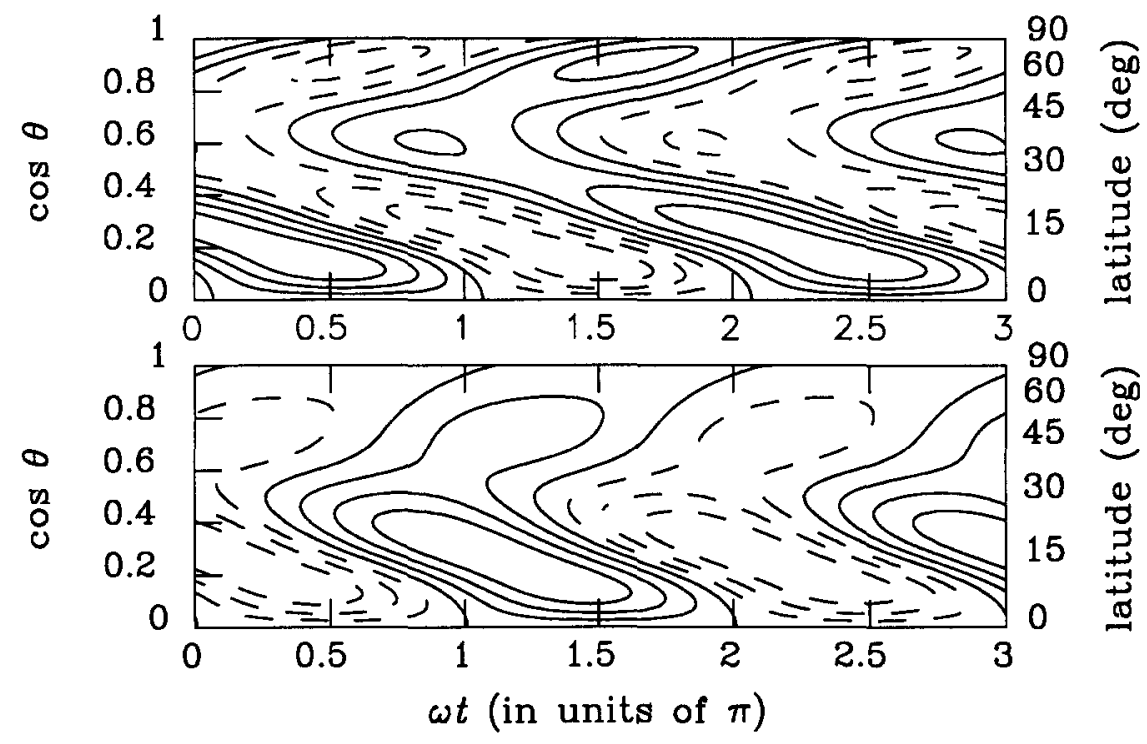

Figure 4: Generalized butterfly diagram for the $B_{r}$-component (upper panel) and for the $B_{\phi}$-component at some reference depth $r=0.9 R$ and $r=0.85 R$, respectively. In both cases a weighted radial average is displayed using a Gaussian with a width of $0.1 R$. The dashed lines refer to negative polarities.

\section{COMPARISON WITH OBSERVATIONS}

We first discuss the toroidal field depicted in the lower panel of Figure 4. Most of the flux is concentrated at low latitudes with a maximum at about $15^{\circ}$. This is in good agreement with the form of the traditional butterfy diagram, and also with synoptic charts of total magnetic flux (see e.g. in $/ 13 /$, Fig.7b). This 
feature of flux concentration is partly due to the presence of the $\beta$ and $\gamma$ terms in Eq.(2). There is also a poleward migrating branch of toroidal field, which is not only due to the effect of latitudinal differential rotation $/ 23 /$, but also because there are two different radial gradients in $\Omega$ (see /12/). Martin and Harvey /16/ found that active regions occuring at higher latitudes have much shorter lifetimes than those at lower latitudes. Their result is in accordance with Figure 3, showing that fields in higher latitudes are situated closer to the surface so that enhanced buoyancy in these layers may be the cause of their short lifetimes. This so-called polar branch is even more pronounced in the $B_{r}$-component of the magnetic field (upper panel). It is hard to see this branch on the original Mt.Wilson/Kitt Peak magnetograms. However, after a "mode cleaning", i.e. removing all odd modes and those with spherical harmonic degree higher than 13 , Stenflo /18/ obtained a generalized butterfly diagram showing this polar branch very clearly (his Fig.4). The separation into two branches starts at about sunspot maximum of the preceeding cycle. A similar branching can be seen in the synoptic chart of the coronal green line /19/. Makarov et al. /12/ presented a combined butterfly diagram showing the latitudinal variation of sunspot activity and faculae. Comparing these results with Stenflo's $B_{r}$-map, there appears a small phase shift: polar activity is first seen in faculae, then in the magnetograms, and finally in the coronal green line. This may be understood as a simultaneous vertical migration of field, as is expected from the dynamo model (see Fig.3). A very important observation for testing dynamo models is the time of reversal of the polar magnetic field. The polar field changes from minus to plus slightly after sunspot maximum, when $B_{\phi}>0$ at lower latitudes (and from plus to minus, when $\left.B_{\phi}<0\right) / 14 /$. This is in good agreement with our models, which display a polar field reversal from minus to plus at $\omega t=1.5 \pi$, somewhat after the positive maximum of $B_{\phi}$.

Finally, we studied the time dependent part of the resulting toroidal Lorentz force (Fig.5), which is presumably driving the so-called torsional oscillations $/ 20,21 /$. Synoptic charts of the torsional oscillation pattern /13/ show two maxima along sloping lines connecting pole and equator, similar as in Fig.5. A high latitude peak at $60^{\circ}$ appeared in 1970, i.e. at about sunspot maximum. This time corresponds in our model to $\omega t=1.3 \pi$. The next peak occured around 1977 at about $25^{\circ}$, slightly after sunspot minimum, corresponding to $\omega t \approx 1.8 \pi$ in the model.

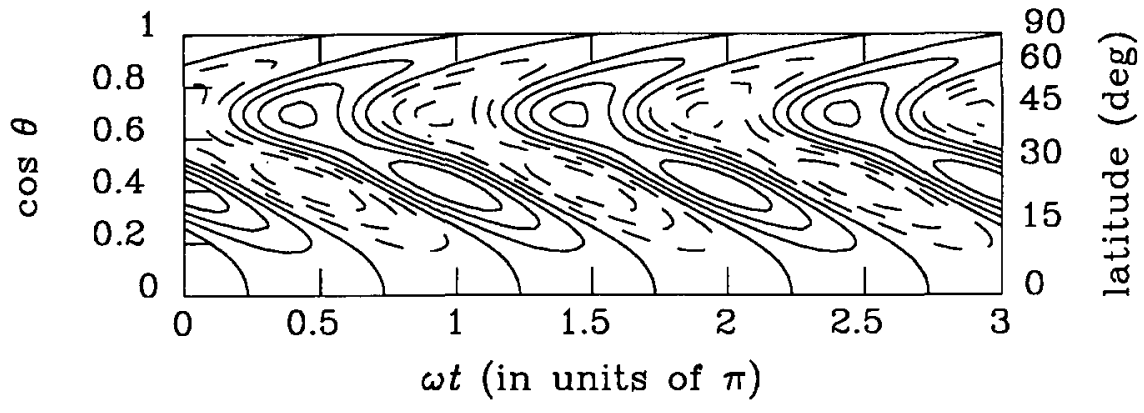

Figure 5: The latitudinal variation of the cyclic part of the Lorentz force.

We conclude that, taking the results of helioseismology and mixing length concept fully into account, it is possible to construct more realistic dynamo models which show good agreement with the observed mean solar magnetic field. For further investigations, it would be of interest to see whether this agreement persists when nonlinear feed-back is taken into account and when the equation of mean motion and the induction equation are solved self-consistently. Also the stability of these solutions and the existance of "mixed parities" /22/ remains to be investigated for solar type models.

\section{REFERENCES}

1. M. Steenbeck and F. Krause, Zur Dynamotheorie stellarer und planetarer Magnetfelder, I. Berechnung sonnenähnlicher Wechselfeldgeneratoren, Astron. Nachr. 291, 49 (1969)

2. M. Stix, Differential Rotation and the Solar Dynamo, Astron. Astrophys. 47, 243 (1976)

3. H. Yoshimura, Phase relation between the poloidal and toroidal solar-cycle general magnetic fields and location of the origin of the surface magnetic fields, Solar Phys. 50, 3 (1976)

4. M. Steenbeck, F. Krause, and K.-H. Rädler, Berechnung der mittleren Lorentz/Feldstärke $\langle\boldsymbol{u} \times \boldsymbol{B}\rangle$ für ein elektrisch leitendes Medium in turbulenter, durch Coriolis-Kräfte beeinflußter Bewegung, $Z$. Naturforsch. 21a, 369 (1966) 
5. P.H. Roberts and A.M. Soward, A unified approach to mean-field electrodynamics, Astron. Nachr. 296, 49 (1975)

6. T. M. Brown, J. Christensen-Dalsgaard, W. A. Dziembowski, P. Goode, D. O. Gough, and C. A. Morrow, Inferring the Sun's internal angular velocity from observed p-mode frequency splittings, submitted to Astrophys. J. (1988)

7. T.L. Duvall Jr and J.W. Harvey, Rotational frequency splitting of solar oscillations, Nature 310, 19 (1984)

8. F. Hill, The equatorial rotation rate in the solar convection zone, in: The Internal Solar Angular Velocity, eds. B.R. Durney and S. Sofia, D. Reidel, Dordrecht, p. 45, 1987.

9. F. Hill, D.M. Rust, and T. Appourchaux, Rotation in the solar convection zone inferred from FabryPerot observations of the 5-min oscillations, in: Advances in Helio- and Asteroseismology, eds. J. Christensen-Dalsgaard and S. Frandsen, D. Reidel Dordrecht, p. 49, 1988.

10. F. Krause, Eine Lösung des Dynamoproblems auf der Grundlage einer linearen Theorie der magnetohydrodynamischen Turbulenz, Habilitationsschrift, Univ. Jena 1967.

11. F. Krause and K.-H. Rädler, Mean-Field Magnetohydrodynamics and Dynamo Theory, AkademieVerlag, Berlin 1980.

12. V.I. Makarov, A.A. Ruzmaikin, and S.V. Starchenko, Magnetic waves of solar activity, Solar Phys. 111,267 (1988)

13. H.B. Snodgrass and R. Howard, Torsional oscillations of the Sun, Science 228, 945 (1985)

14. V.I. Makarov, M.P. Fatianov, and K.R. Sivaraman, Poleward migration of the magnetic neutral line and the reversal of the polar fields on the Sun, Solar Phys. 85, 215 (1983)

15. D. Gough, What causes the solar cycle?, Nature 319, 263 (1986)

16. S.F. Martin and K.L. Harvey, Ephemeral active regions during solar minimum, Solar Phys. 64, 93 (1983)

17. I. Tuominen and H. Virtanen, Variation of the solar rotation at the surface and deep in the convection zone, this issue.

18. J.O. Stenflo, Global wave patterns in the Sun's magnetic field, Astrophys. Spa. Sci., in press (1988)

19. J.-L. Leroy and J.-C. Noens, Does the solar activity cycle extend over more then an 11-year period? Astron. Astrophys. 120, L1 (1983)

20. R. Howard and B.J. LaBonte, The Sun is observed to be a torsional oscillator with a period of 11 years, Astrophys. J. 239, L33 (1980)

21. G. Rüdiger, I. Tuominen, F. Krause, and H. Virtanen, Dynamo-generated flows in the Sun, I. Foundations and first results, Astron. Astrophys. 166, 306 (1986)

22. A. Brandenburg, F. Krause, R. Meinel, D. Moss, and I. Tuominen, The stability of nonlinear dynamos and the limited rôle of kinematic growth rates, submitted to Astron. Astrophys. (1988)

23. H. Yoshimura, A model of the solar cycle driven by the dynamo action of the global convection in the solar convection zone, Astrophys. J. Suupl. 29, 467 (1975) 